\section{Nanostructured Lithium Sulfide/Silicon Electrode Design Quadruples Theoretical Specific Energy of Rechargeable Batteries}

With recent advances in high-capacity anodes, the limiting factor in producing rechargeable batteries with a high specific energy (for use in electric vehicles and portable electronics) now stems from the relatively low specific capacity of the corresponding cathodes. While sulfur-based cathodes in combination with lithium anodes have the potential to overcome these capacity limitations, the use of elemental $\mathrm{Li}$ as the anode leads to serious safety concerns- $-\mathrm{Li}$ dendrites form during cycling and can penetrate the thin polymer layer separating the two electrodes, leading to short circuits and potential explosions. Y. Yang, A.T. McDowell, A. Jackson, and colleagues at Stanford University, however, combat these issues by pairing sulfur's lithiated counterpart $\left(\mathrm{Li}_{2} \mathrm{~S}\right)$ in the cathode with a high capacity, low potential silicon nanowire anode to create a novel nanostructured rechargeable battery with a theoretical specific energy four times that of existing $\mathrm{LiCoO}_{2} /$ graphite or $\mathrm{LiFePO}_{4} /$ graphite systems.

As described in the April 14 issue of Nano Letters (DOI: 10.1021/nl100504q; p. 1486), on the cathode side of the battery depicted in Figure 1, the researchers first diffuse sulfur into the sub- $5 \mathrm{~nm}$ pores of ordered mesoporous carbon and then convert it into $\mathrm{Li}_{2} \mathrm{~S}$ by a reaction with n-butyllithium. The ordered mesoporous carbon consists of hexagonally arranged 7-8-nm thick nanorods around 3-4 nm pores. The interconnected carbon rods act as conductive pathways, providing electronic access to the insulating $\mathrm{Li}_{2} \mathrm{~S}$ trapped within the pores while the submicrometer

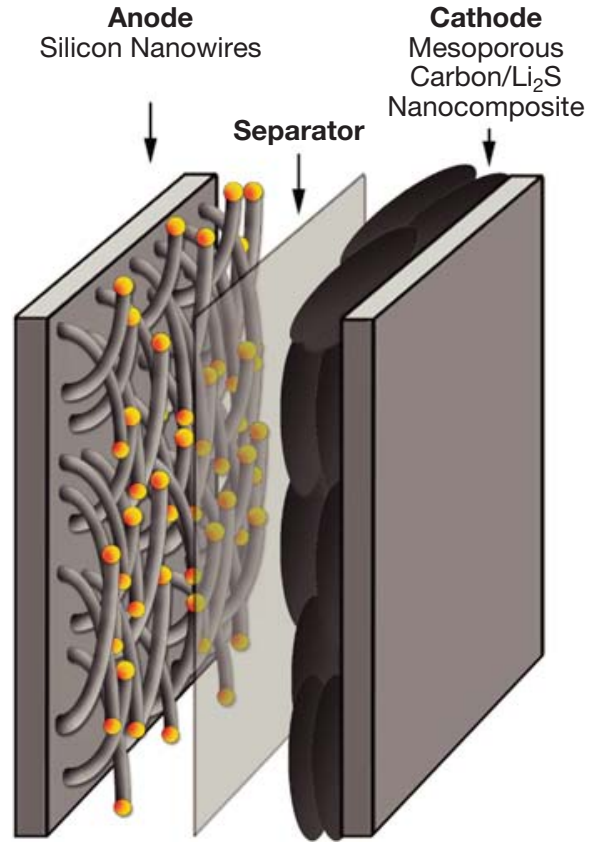

Figure 1. Schematic of the $\mathrm{Li}_{2} \mathrm{~S} / \mathrm{Si}$ battery structure: The cathode contains lithium-sulfide incorporated within the pores of ordered mesoporous carbon, and the anode consists of silicon nanowires grown onto stainless steel substrates using the vapor-liquid-solid method. Reproduced with permission from Nano Lett. 10(4)(2010) 1486; DOI: 10.1021/nl100504q. C2010 American Chemical Society.

size of the carbon particles shortens Li diffusion paths-this design overcomes the slow kinetics (and poor electronic conductivity) inherent to $\mathrm{Li}_{2} \mathrm{~S}$-based cathodes. On the anode side, silicon nanowires are grown by the vapor-liquid-solid (VLS) method onto a stainless steel substrate. Silicon is known to have a very high theoretical capacity (4212 $\mathrm{mAh} / \mathrm{g}$ ) and low potential $\left(\sim 0.3 \mathrm{~V}\right.$ versus $\left.\mathrm{Li} / \mathrm{Li}^{+}\right)$, and unlike previous Si-based electrodes, the nanowire architecture allows for the requisite $400 \%$ volume change associated with the insertion and extraction of Li without disintegration or significant capacity fading.

The resulting higher capacity and lower overpotential of $\mathrm{Li}_{2} \mathrm{~S} /$ mesoporous carbon as compared to $\mathrm{Li}_{2} \mathrm{~S} /$ macroporous carbon observed by the researchers clearly demonstrate the importance of size, morphology, and structure of the porous carbon on the corresponding electrochemical performance of the composite cathode. When examining the performance of the full battery cell at a lowered current rate (corresponding to an eight-hour discharge time), the researchers can thus far achieve an initial discharge specific energy of $630 \mathrm{Wh}$ per $\mathrm{kg}$ of active electrode material (as compared to the theoretical calculation of $1550 \mathrm{Wh} / \mathrm{kg}$ ). If the total electrode mass is considered, this corresponds to a specific energy of $\sim 349 \mathrm{Wh} / \mathrm{kg}$, which is similar to the $335 \mathrm{Wh} / \mathrm{kg}$ provided by commercial Li-ion batteries. Moreover, the researchers project that "the specific energy could reach $\sim 600 \mathrm{Wh} / \mathrm{kg}$ considering the total electrode mass with further optimization of the battery." They also said that "utilization of better electrolytes and surface modification of the electrodes are necessary to overcome the cycling behavior and capacity decay issues associated with their current design," thereby enabling competition with well-developed Li-ion battery systems.

TifFAny D. ZiEbeLL

\section{Sol-Gel Electrophoretic Deposition Process Improves Dye-Sensitized Solar Cells}

Dye-sensitized solar cells (DSSCs) hold significant promise as a lower cost alternative to conventional silicon photovoltaics. Their ability to be fabricated using inexpensive techniques on flexible plastic substrates has attracted considerable research interest since they were first demonstrated almost 20 years ago. However, their efficiencies are limited by electron losses in the anode. Now A. Zaban, L. Grinis, and their colleagues at Bar-Ilan University in Ramat Gan, Israel have demonstrated a new low-temperature coating technique using sol-gel processing and electro- phoretic deposition (EPD) that partially overcomes this limitation. They reported their findings in the January 22 issue of Advanced Functional Materials (DOI: 10.1002/adfm.200901717; p. 282).

DSSCs are based on photosensitive dyes covalently bonded to a mesoporous scaffolding of $\mathrm{TiO}_{2}$ nanoparticles. During operation, photons from sunlight produce photoelectrons in the dye, which are then injected into the nanoparticles for transport to a conducting substrate while the dye is reduced by an electrolyte permeating the system. Electrons must travel through tens or hundreds of nanoparticles to reach the substrate, making good inter-particle electrical connections and low recombination rates with holes in the electrolyte critical for efficient operation. Numerous approaches to overcoming these problems have been investigated involving coatings on the scaffolding, but many of them require high-temperature sintering, which would prohibit fabrication on flexible plastic substrates.

To address this problem, the researchers coated the nanoparticles using a lowtemperature sol-gel EPD process based on metal alkoxides carried out from dilute alcohol solutions containing iodine, acetone, and water, at low current densities $\left(25-100 \mu \mathrm{A} / \mathrm{cm}^{2}\right)$. This resulted in thin, amorphous coatings of titania, magnesia, or niobia on the mesoporous $\mathrm{TiO}_{2}$, which 